\title{
Unsupervised Learning Based Emission-aware Uplink Resource Allocation Scheme for Non-Orthogonal Multiple Access Systems
}

\author{
Muhammad Ali Jamshed, Student Member, IEEE, Fabien Héliot, Member, IEEE, \\ and Tim W.C. Brown, Member, IEEE
}

\begin{abstract}
The densification of wireless infrastructure to meet ever-increasing quality of service $(\mathrm{QoS})$ demands, and the evergrowing number of wireless devices may lead to higher levels of electromagnetic field (EMF) exposure in the environment, in the $5 \mathrm{G}$ era. The possible long term health effects related to the EMF radiation are still an open debate and requires attention. Therefore, in this paper, we propose a novel EMFaware resource allocation scheme based on the power domain non-orthogonal multiple access (PD-NOMA) and machine learning (ML) technologies for reducing the EMF exposure in the uplink of cellular systems. More specifically, we use the K-means approach (an unsupervised ML approach) to create clusters of users to be allocated together and to then strategically group and assign them on the subcarriers, based on their associated channel properties. Finding the best number of clusters in the PD-NOMA environment is a key challenge, and in this paper, we have used the elbow method in conjunction with the F-test method to effectively control the maximum number of users to be allocated at the same time per subcarrier. We have also derived an EMF-aware power allocation by formulating and solving a convex optimization problem. Based on the simulation results, our proposed ML-based strategy effectively reduces the EMF exposure, in comparison with the state-of-the-art techniques.
\end{abstract}

Index Terms-Resource allocation, machine learning (ML), non-orthogonal multiple access systems, electromagnetic emission, convex optimization.

\section{INTRODUCTION}

$\mathrm{T}$ HE advancements in the wireless communication industry have revolutionized the way in which societies generate, circulate, receive, and perceive information. It is foreseen that the capacity will have to grow by 1,000 folds to accommodate the ever-growing number of wireless users and internet of things (IoT) devices (augmented reality, wearable sensors, etc.), in the $5 \mathrm{G}$ era [1]. This capacity enhancement will be made possible via an increase in the number of access points (APs). In other words, the number and type of electromagnetic field (EMF) exposure sources in the environment will considerably increase in the $5 \mathrm{G}$ era. Even though there is still

Copyright (c) 2015 IEEE. Personal use of this material is permitted. However, permission to use this material for any other purposes must be obtained from the IEEE by sending a request to pubs-permissions@ieee.org.

This work has been funded by EPSRC under the grant agreement "EP/P005853/1: Electro-magnetic field exposure reduction/avoidance for the next generations of wireless communication systems". We would also like to acknowledge the support of the University of Surrey 5GIC and 6GIC (http://www.surrey.ac.uk/5gic) members for this work. M. A. Jamshed, F. Héliot and T. Brown are with the Institute of Communication Systems (ICS),Home of 5G \& 6G Innovation Centres, University of Surrey, UK. Email: $\{$ m.jamshed, f.heliot, t.brown $@ @$ surrey.ac.uk. no strong evidence related to the short term health effects of EMF exposure, EMF radiation from wireless devices has been classified by the international agency for research on cancer (IARC) and world health organization (WHO) as possibly carcinogenic to humans (group B) [2]. Meanwhile, the growth in EMF radiations in the $5 \mathrm{G}$ era could only increase the potential health hazards (long term) related to EMF [3].

In order to limit the potential health effect of EMF exposure, user equipment (UE) antennas must comply with EMF regulations. For instance, the Federal communications commission (FCC) in the USA provides local specific absorption rate (SAR) guidelines, i.e. for $1 \mathrm{~g}$ of body tissue the maximum SAR value should not exceed $1.6 \mathrm{~W} / \mathrm{kg}$, and similarly, for 10 $\mathrm{g}$ of tissue, it should be less than $2.0 \mathrm{~W} / \mathrm{kg}$ [4]. Meanwhile, at the international level, the restrictions have been defined for both passive (power density (PD)) and active (SAR) EMF exposure by the international commission on non-ionizing radiation protection (ICNIRP) [5]. As per recent ICNIRP recommendations, the whole-body average SAR (i.e. global SAR) should not exceed $0.4 \mathrm{~W} / \mathrm{kg}$. However, based on FCC consumer guidelines; "FCC approval does not indicate the amount of EMF exposure consumers experience during normal use of the device" [6].

The previous generations ( $2 \mathrm{G}-4 \mathrm{G})$ of mobile communications relied on orthogonal multiple access (OMA) schemes, i.e. orthogonal frequency division multiple access (OFDMA), time-division multiple access (TDMA), etc., to perform user multiplexing. However, the non-orthogonal multiple access (NOMA) has emerged as a promising multiplexing approach for the fifth generation of cellular communication technology. There are two main types of NOMA, which are known as power domain-NOMA (PD-NOMA) [7]-[9] and codedomain NOMA (CD-NOMA) (e.g. sparse code multiple access (SCMA) [10], [11]), and in this paper we focus on the former. The PD-NOMA exploits the variation in channel gain between the different users to allocate them on the same subcarrier with different transmission powers; hence enabling the system to schedule more users at the same time [7]. In comparison to OMA, NOMA can handle an increased number of users, hence increasing the spectral efficiency of the system. Moreover, these properties of classic PD-NOMA opens some exciting opportunities to reduce EMF exposure, without compromising on the quality of service (QoS). 


\section{A. Existing Work}

According to our recent literature survey on EMF exposure risk assessment and evaluation mechanism in [12], most of the existing works related to EMF reduction in the uplink of cellular system boils down to SAR reduction. For instance, in [13], the SAR is effectively reduced by placing a metamaterial between the UE and the human head. The works aiming at the minimization of the SAR only cover up the worst-case scenarios but do not take into account the effect of EMF exposure over time. In [14], an EMF-aware scheduling scheme have been proposed for the uplink of OFDMA based systems, which exploits the definition of the dose metric (measurement of SAR over time), to reduce the user EMF exposure in a single cell system, while ensuring QoS. Likewise in [15], a similar approach is used to reduce EMF exposure in a OFDMA based multi-cell environment. Whereas in [16], a cell-selection mechanism is used to minimize the uplink EMF exposure of users in a single cell scenario, while satisfying the downlink throughput requirements of all these users.

As far as PD-NOMA is concerned, most of the existing works have focused on the downlink scenario, whereas a few of them has considered the uplink of PD-NOMA [17], [18]. For instance, in [17], the throughput is enhanced by strategically adjusting the power levels of each user, being allocated to the same subcarrier, while comparing it with traditional OMA scheme. Similarly, the spectral efficiency of a PD-NOMA based system is incremented by using an effective approach to allocate subcarrier and at the same time performing optimal power assignment [18]. Along with efficient power allocation strategies, the performance of PD-NOMA systems highly depends on the optimal user grouping/paring on the same subcarrier. In [19], a dynamic user grouping strategy is proposed, based on the underlying Rayleigh faded channel coefficients, and to increase the detection accuracy at the receiver end by maintaining a minimum interference between the users that share the same subcarrier. The authors in [8] uses an adaptive user pairing methodology to identify a user far from the access point (AP) and then select a user with a better channel condition to group them on a same subcarrier. Recently, the use of machine learning (ML) based clustering algorithms are used to intelligently perform the user grouping for NOMA systems by identifying an appropriate number of clusters [9]. The current literature on clustering algorithms classifies them as hierarchical or partitional algorithms [20]. The hierarchical clustering mechanism consists of a set of nested clusters organized as trees, whereas the partitional algorithms cluster the data in disjoint subsets [21].

\section{B. Motivation and Contributions}

In comparison to OMA, the users having a difference in the power levels can be multiplexed on a same subcarrier using PD-NOMA mechanism. To reduce the interference between the users sharing the same subcarrier, it is highly recommended that users have a low channel correlation. Therefore, the selection of effective user clustering techniques plays a critical role in realizing the true potential of a PD-NOMA system. The combinatorial feature of user grouping/clustering motivates the need of using ML algorithms especially for large systems. The learning features of the ML algorithms provides an additive advantage to cluster the users in different groups based on their associated channel properties, in comparison to suboptimal algorithms, i.e. matching theory [22] etc. The selection of the clustering algorithms mainly depends upon the distribution of the data set present in the feature space. A clustering learner can be designed by intelligently partitioning the data set in the feature space using a measurement function, which is defined as the unsupervised machine learning approach.

In this paper, we propose a novel EMF-aware scheduling scheme that leverage on PD-NOMA and unsupervised ML for reducing further than other existing scheduling schemes the uplink exposure of cellular system users, while complying with QoS requirements. We use ML to design an efficient user grouping method that help to ensure a successful decoding at the receiver end. We have first clustered the users by using the K-means [9] clustering algorithm, and then the users have been efficiently grouped and allocated on the subcarriers to reduce their exposure. The K-means approach is based on a partitional clustering strategy that groups the data in a number of clusters specified by the user. To the best of our knowledge, there is not yet any available work in the literature that have leveraged on the unsupervised ML concept, to minimize the EMF exposure of cellular systems relying on the PD-NOMA technology. Our main contributions to this paper are outlined as follows:

- In comparison with [14], we design a multiuser EMFaware scheduling relying on the PD-NOMA technology instead of the classic OFDMA technology in [14], which makes the design more challenging, e.g. user grouping, but provide further benefits in terms of exposure reduction. A preliminary version of this work can be found in [23]. In comparison with [23], this work provides more robust and efficient methods for grouping the users (based on ML) and allocating these users on the subcarriers. It also incorporates the effect of successive interference cancellation (SIC) in the allocation process by taking into account a minimum interference constraint between the users sharing the same subcarrier to make our approach more suitable for realistic scenarios.

- Similar to [9] we have used the K-means approach to cluster the users in different groups. However, contrary to [9] and for the first time, we have used the elbow method in conjunction with the F-test method to find the best number of clusters for a given channel condition. Moreover, our proposed user clustering algorithm provides a better control over the number of users per subcarrier and more efficient utilization of the bandwidth than [9]. Furthermore, this work also provides a mathematical definition of calculating the F-test value for a given number of clusters, formed using K-means, while relying on the associate channel properties of each user. Lastly, we perform power allocation in a EMF-aware manner.

- The performance of the proposed strategy have been validated by using Monte Carlo types of simulations. 
We have compared our proposed scheme against a similar OFDMA-based EMF-aware multiuser scheduling scheme, a spectral efficient PD-NOMA scheme, and the greedy subcarrier allocation scheme and have shown that our new EMF-aware PD-NOMA scheme can effectively reduce the EMF exposure. Our novel scheme reduces the EMF exposure by at least $28 \%$ in comparison to [14] and by at least 2 and 3 order of magnitude, while comparing with [18] and the greedy scheme.

- We have also validated our results by the SAR values calculated using the simulation scenarios build in CST STUDIO SUITE 2018 based on IEEE recommendations. Moreover, we have designed a planar inverted-F antenna (PIFA) equipped with a single transmit antenna to mimic the characteristics of a mobile handset. This type of analysis also validates the superiority of using PIFAs in mobile handsets.

\section{Structure of the paper}

The remainder of the paper is organized as follows: in Section II a detailed explanation of the system model is provided, present the scenario of interest and mathematical framework that have been considered to design our EMFaware PD-NOMA proposed scheme. Section III covers the aspects of the novel proposed ML-based user grouping and resource allocation mechanism. Meanwhile, Section IV provides insights about our EMF-aware power allocation strategy. Section $\mathrm{V}$ presents the simulation results along with their relevant observations. Finally, Section VI concludes the paper. The list of symbols with their meanings, used throughout the paper are listed in Table I.

\section{EMF-AWARE PD-NOMA FRAMEWORK}

\section{A. System Model}

As illustrated in Fig. 1 and without loss of generality, we consider a single-cell communication system where $K$ users rely on single antenna UEs to communicate with a base station (BS) by using the PD-NOMA multiplexing strategy. The bandwidth of the system, $W$, is divided into $N$ subcarriers, and since we consider a NOMA scenario, more than one user can be assigned to the same subcarrier. Assuming that $K_{n, t}$ users are assigned to the $n^{\text {th }}$ subcarrier at time slot $t$, then the corresponding received signal at the BS can be expressed as:

$$
y_{n, t}=\sum_{k=1}^{K_{n, t}} \sqrt{p_{k, n, t} g_{k, n, t}} x_{k, n, t}+z_{n, t},
$$

where $x_{k, n, t}$ is the information signal of $k^{\text {th }} \mathrm{UE}$ on subcarrier $n$ at time slot $t, p_{k, n, t}$ is the transmit power of the $k^{\text {th }} \mathrm{UE}$ on subcarrier $n$ at time slot $t, g_{k, n, t}$ is the channel gain between the $k^{\text {th }} \mathrm{UE}$ and the BS on subcarrier $n$ at time slot $t$, and $z_{n, t}$ models the additive white Gaussian noise (AWGN), having a zero mean and a variance of $\sigma^{2}$, on subcarrier $n$ at time slot $t$. Since in NOMA the data of different users can be multiplexed on the same subcarrier, interference occurs. The total interference experienced by the $k^{\text {th }}$ user of subcarrier $n$ can be expressed in the general case as:
TABLE I

NOTATIONS WITH DEFINITIONS USED THROUGHOUT THE PAPER

\begin{tabular}{|l||l|}
\hline \hline \multicolumn{1}{|c|}{ Symbol } & \multicolumn{1}{c|}{ Meaning } \\
\hline \hline$R$ & Cellular radius \\
\hline$\beta$ & Conductivity \\
\hline$\alpha$ & Subcarrier index \\
\hline$\sigma^{2}$ & Noise power density per subcarrier \\
\hline$M_{d}$ & Mass density \\
\hline$K$ & Total number of users \\
\hline$T$ & Total number of time slots \\
\hline$S$ & Total subcarrier allocated over $T$ \\
\hline$g_{n}$ & Gain of user $n$ \\
\hline$W$ & Total bandwidth \\
\hline$E_{f}$ & Electric field intensity \\
\hline$D_{k}$ & Path loss \\
\hline$P_{k}^{\max }$ & Maximum transmit power \\
\hline $\bar{p}_{k}$ & Signaling power of $k^{t h}$ user \\
\hline$\tau$ & Duration of time slot $t$ \\
\hline$a$ & CQI bits \\
\hline$\zeta$ & Reference threshold \\
\hline$M$ & Number of clusters \\
\hline$z_{n}$ & Gaussian noise \\
\hline$P_{o}$ & Received signal power \\
\hline $\mathrm{SAR}_{k}^{a}$ & $k^{t h}$ user SAR for cheek position \\
\hline $\mathrm{SAR}_{k}^{b}$ & $k^{t h}$ user SAR for tilt position \\
\hline$B r$ & Required number of bits \\
\hline$K_{n, t}$ & Maximum number of users on $\alpha$ \\
\hline \hline & \multicolumn{2}{|l|}{} \\
\hline
\end{tabular}

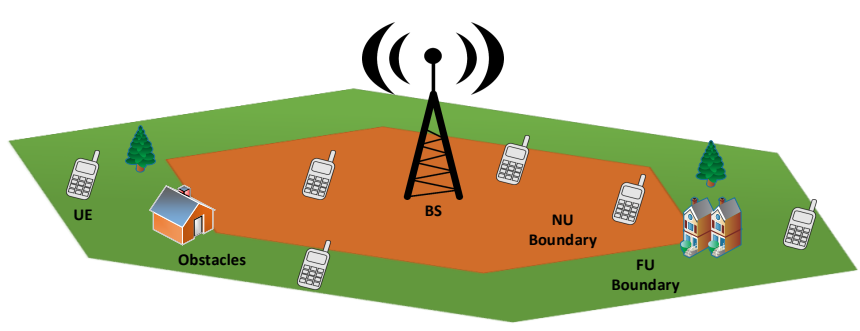

Fig. 1. An illustration of a single cell system with multiple users.

$$
\bar{I}_{k, n, t}=\sum_{l=1, l \neq k}^{K_{n, t}} p_{l, n, t} g_{l, n, t} .
$$

In PD-NOMA, the SIC process is usually employed at the receiver (i.e. BS in our case) to decode the data of the different users that are multiplexed together. It is well-known from multiple-input-multiple-output (MIMO) detection, for which SIC was originally designed [24], that the order of the detection has an impact on the performance of the process. Traditionally, the data being sent over the best channel gain are decoded first while the ones transmitted over the worst channel gain are decoded last in SIC-based MIMO detection. In the uplink of PD-NOMA, this rule translates as the user having the best received power at the BS (i.e. 
$\left.p_{\pi(1), n, t} g_{\pi(1), n, t}\right)$ being decoded first, while the one with the worst power at the BS (i.e. $p_{\pi\left(K_{n, t}\right), n, t} g_{\pi\left(K_{n, t}\right), n, t}$ ) being decoded last, where $\pi$ is an index permutation vector based on the SIC decoding order, such that $\pi(1)=\operatorname{argmax}_{k} p_{k, n, t} g_{k, n, t}$, $\pi\left(K_{n, t}\right)=\operatorname{argmin}_{k} p_{k, n, t} g_{k, n, t}$, and $p_{\pi(1), n, t} g_{\pi(1), n, t} \geq$ $p_{\pi(2), n, t} g_{\pi(2), n, t} \geq \ldots \geq p_{\pi\left(K_{n, t}\right), n, t} g_{\pi\left(K_{n, t}\right), n, t}$ on any given subcarrier $n$ of time slot $t$. Consequently, the power allocation at the UE will have an impact on the performance of the SIC process, i.e. on its ability to dissociate between the users. The multiplexing strategy of PD-NOMA is based on the fact that SIC should be able to decode the multiplexed signal successfully [25]. In order to take this into account in our model, we consider that this can be achieved for any user $k$ on subcarrier $n$ of time slot $t$ if its received signal to residual interference ratio is greater than or equal to a reference threshold $\zeta$, i.e.:

$$
p_{k, n, t} g_{k, n, t} / I_{k, n, t} \geq \zeta
$$

$\zeta \geq 1$, where:

$$
I_{k, n, t}=\sum_{l=\pi^{-1}(k)+1}^{K_{n, t}} p_{\pi(l), n, t} g_{\pi(l), n, t} .
$$

In (4), $\pi^{-1}(k)$ returns the index of $k$ in the permutation vector $\pi$, e.g. for instance if $\pi(1)=5$, then $\pi^{-1}(5)=1$, i.e. the index of 5 in $\pi$ is 1 . Consequently, when the SIC process is always successful, or in other words, when the constraint in (3) is always met, then $I_{\pi\left(K_{n, t}\right)}=0, \forall n, t$. Based on equations (1), (4) and Shannon theory, the total number of bits transmitted by user $k$ on subcarrier $n$ over a time slot $t$ of duration $\tau$ can be calculated by using the following expression:

$$
b_{k, n, t(\boldsymbol{\alpha}, \boldsymbol{p})}=w \tau \alpha_{k, n, t} \log _{2}\left(1+\frac{p_{k, n, t} g_{k, n, t}}{\sigma^{2}+I_{k, n, t}}\right),
$$

where, $w=W / N$ is the bandwidth of each subcarrier, $\alpha_{k, n, t}$ is a subcarrier allocation index, i.e. $\alpha_{k, n, t}=1$ if user $k$ is allocated to subcarrier $n$ at time slot $t$ or 0 otherwise, $\mathbf{p}=\left[p_{1,1,1} ; \ldots ; p_{K, N, 1} ; p_{1,1,2} ; \ldots ; p_{K, N, T}\right] \geq 0$, and $\boldsymbol{\alpha}=$ $\left[\alpha_{1,1,1} ; \ldots ; \alpha_{k, N, 1} ; \alpha_{1,1,2} ; \ldots ; \alpha_{K, N, T}\right]$.

As far as the individual exposure of user $k$ in the uplink is concerned, it can be expressed, based on [26], as:

$$
E_{k}(\boldsymbol{\alpha}, \boldsymbol{p})=\frac{\mathrm{SAR}_{k}}{T P^{\mathrm{ref}}} \tau\left(\bar{p}_{k}(T)+\sum_{t=1}^{T} \sum_{n=1}^{N} \alpha_{k, n, t} p_{k, n, t}\right)
$$

here, $T$ is the number of time slots, $P^{\text {ref }}$ is defined at the reference incident power at which the SAR of the $k^{\text {th }} \mathrm{UE}$ has been calculated. The SAR metric is generally used to measure the EMF exposure in the near-field of an antenna operating below $10 \mathrm{GHz}$. It can be categorized as average or organ-specific SAR, e.g. head, hand, etc [12], and it is usually expressed as:

$$
\mathrm{SAR}=\frac{\beta \times E_{f}^{2}}{M_{d}} \quad(\mathrm{~W} / \mathrm{kg})
$$

where $\beta, M_{d}$ accounts for the conductivity and the mass density of the exposed object/tissues, respectively, and the $E_{f}$ indicates the electric field. Note that the FCC enforces a limit of $1.6 \mathrm{~W} / \mathrm{kg}$ for one gram of body mass for the SAR of UEs [27]. Meanwhile, $\bar{p}_{k}(T)$ in (6) represents the signaling power used by the UE of user $k$ for setting up its data transmission over $T$ time slots; it can mathematically be expressed, based on [28], as:

$$
\bar{p}_{k}(T)=\min \left(P_{k}^{\max }, P_{o} D_{k} \max (a T / 4,1)\right),
$$

where, $P_{k}^{\max }$ is the $k^{\text {th }}$ UE maximum transmit power, $D_{k}$ denotes the path loss experienced by user $k, P_{o}$ denotes the received signal power threshold at the $\mathrm{BS}$, and $a$ denotes the number of channel quality index (CQI) bits used to measure the channel quality.

\section{B. Problem Formulation}

The main aim of our work being to design an EMFaware scheduling scheme for reducing the exposure when considering QoS and PD-NOMA with SIC, we first formulate its mathematical framework in the form of an optimization problem, as follows:

$$
\underset{\boldsymbol{p}, \boldsymbol{\alpha}}{\operatorname{minimize}} E(\boldsymbol{\alpha}, \boldsymbol{p})=\sum_{i=1}^{K} E_{k}(\boldsymbol{\alpha}, \boldsymbol{p}),
$$

subject to:

$$
\begin{aligned}
& C_{1}: \sum_{t=1}^{T} \sum_{n=1}^{N} b_{k, n, t}(\boldsymbol{\alpha}, \boldsymbol{p})=B r_{k}, \forall k \\
& C_{2}: \sum_{n=1}^{N} \alpha_{k, n, t} p_{k, n, t} \leq P_{k}^{\max } \quad \forall k, \forall t, \\
& C_{3}: \alpha_{k, n, t}\left(p_{k, n, t} g_{k, n, t} / I_{k, n, t}\right) \geq \zeta \quad \forall k, \forall n, \forall t \\
& C_{4}: \sum_{k=1}^{K} \alpha_{k, n, t} \leq K_{n, t} \quad \forall n, \forall t .
\end{aligned}
$$

The objective function $E(\alpha, \mathbf{p})$ in (9) represents the total uplink exposure experienced by the $K$ users of the system over $T$ time slots. Meanwhile, the constraints in (10) can be defined as follows:

- The constraint $C_{1}$ is a QoS constraint that ensures that each user transmits its required number of bits, i.e. $B r_{k}$ in (10a).

- The constraint $C_{2}$ is a transmit power constraint; it reflects that the transmit power of each UE is limited by a maximum value, i.e. $P_{k}^{\max }$ in $(10 \mathrm{~b})$, for each time slot.

- The constraint $C_{3}$ is related to the SIC process at the BS; if (10c) is met then it implies that SIC can remove some interference from the received signal of user $k$.

- The constraint $C_{4}$ is related to NOMA; it reflects that the number of users that can be grouped on subcarrier $n$ at any time slot $t$ is at most $K_{n, t}$, when using NOMA.

It may be noted that our optimization problem, which is defined in (9) and (10), is non-convex given the binary nature of $\alpha_{k, n, t}$ [29], and given that the equality constraint $C_{1}$ is non-affine. In order to solve this problem efficiently, we first need to overcome its binary nature by applying a standard relaxation mechanism. Indeed, we consider, as for instance in 
[30], a sequential subcarrier and power assignment, i.e. by first finding $\boldsymbol{\alpha}$ for a fixed $\mathbf{p}$ and then finding $\mathbf{p}$ for a fixed $\boldsymbol{\alpha}$. A change of variable needs also to be apply to (9) and (10) to overcome the non-affinity of $C_{1}$ and makes them convex when $\boldsymbol{\alpha}$ is fixed.

These two aspects are further detailed in the two following sections. Firstly, in Section III, we explain our novel ML based grouping and subcarrier allocation method that intelligently provides a complete control over the number of users grouped on the same subcarrier, and reduces the complexity of SIC at the receiver end by grouping the users on a subcarrier $n$ having a minimum interference. Secondly, in Section IV, we present our power allocation strategy, which is integrated with our ML based subcarrier allocation. It optimizes the transmit power level of each user to ensure that their QoS is meet. It may be noted that, as in [14], we assume that the channel state information (CSI) of the $K$ users over $T$ time slots can be predicted in advance by the BS through uplink pilot signals.

\section{MACHINE LEARNing BASED USER GROUPING/SUBCARRIER ALLOCATION}

The PD-NOMA scheme allows to increase the spectral efficiency of communication systems [7] by efficiently demultiplexing multiple users sharing the same subcarrier based on their received power levels. Its effectiveness heavily depends on the capability of the receiver at differentiating between different levels of user received powers, which itself depends on the selection/grouping of the users that are multiplexed together. For instance, in the uplink of a cellular system, the location of the users (near user (NU) and far user (FU) in Fig. 1) can be exploited for grouping the users (i.e. NU and FU are likely to have quite different received power levels at the BS due to their large difference in path-loss).

When it comes to user grouping, the use of ML algorithms significantly reduces the complexity and increases the probability of converging to an optimal solution as compared to nonML algorithms [31]. For instance, K-means clustering, which is one of the simplest and popular types of unsupervised ML algorithms, has proved to be an effective method for grouping users in [9]. The K-means algorithm clusters data into several groups/clusters of data, where the desired number of clusters has to be specified prior to running the algorithm. Hence, finding the best number of clusters for achieving a particular goal is an important part of the clustering process [32]. A general rule of thumb for selecting this number is to chose it to be relatively small in comparison with the size of the data set to be clustered (i.e. $g_{k, n, t} \forall k, n, t$, in our case). In the context of PD-NOMA, the authors in [9] used a simplified version of the elbow method [33] to set their desired number of clusters. This version of the elbow method uses the K-means method to calculate the average internal cluster distance for a specified range of desired cluster numbers [9].

Here, similar to [9], we rely on the K-means method for clustering the user, but we use the F-test method [34] in conjunction with the elbow method to adjust our required number of clusters per subcarrier and time slot. For a given number of clusters $M$, the F-test calculates the ratio of the

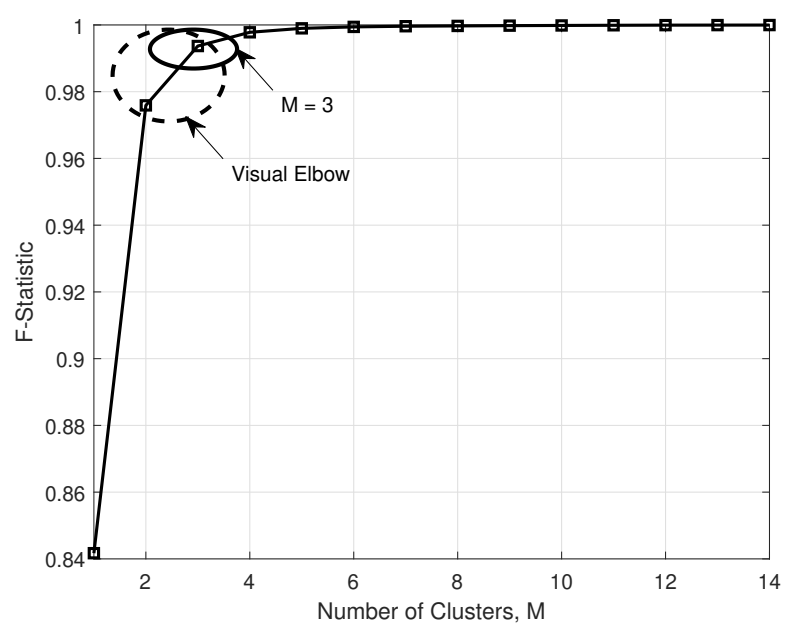

Fig. 2. F-test statistic in (11) as a function of $M$ for $K=100, N=1$, and $T=1$.

variability between the different clusters (i.e. formed by using $\mathrm{K}$-means) to the total variability, which is the sum of the variability between the different clusters and the variability within the clusters, as follows:

$$
\begin{array}{r}
F_{n, t}(M)=\frac{\sum_{m=1}^{M} K_{m}\left(\bar{g}_{m, n, t}-\bar{g}_{n, t}\right)^{2} /(M-1)}{\left[\sum_{m=1}^{M} \sum_{j=1}^{K_{m}}\left(g_{m(j), n, t}-\bar{g}_{m, n, t}\right)^{2} /(K-M)\right.} \\
\left.+\sum_{m=1}^{M} K_{m}\left(\bar{g}_{m, n, t}-\bar{g}_{n, t}\right)^{2} /(M-1)\right],
\end{array}
$$

for each subcarrier of each time slot. In (11), $K_{m}$ is the number of users in the $m^{\text {th }}$ cluster and $\bar{g}_{n, t}=\sum_{k=1}^{K} g_{k, n, t} / K$ is the mean of $g_{k, n, t}$ over the $K$ users, $\forall n, t$. Moreover, $\bar{g}_{m, n, t}=\sum_{j=1}^{K_{m}} g_{m(j), n, t} / K_{m}$ is the mean of $g_{k, n, t}$ over the $K_{m}$ users belonging to the $m^{\text {th }}$ cluster, $\forall n, t$, where $g_{m(j), n, t}$ represents the channel gain of the $j^{\text {th }}$ user belonging to the $m^{\text {th }}$ cluster, $\forall n, t$. Figure 2 illustrates $F$-test results as a function of the number of clusters $M$ when running the K-means algorithm [9] for each of these values of $M$ (i.e. $M=1$ to 14 ) for $K=100$ over one subcarrier/time slot (i.e. $N=T=1$ ) and assuming that $g_{k, 1,1}$ follows a Rayleigh distribution (by using the path-loss model defined in [35]), $\forall k$. The results show that the F-test increases as $M$ increases, i.e. the similarities between the channel gains within each clusters and the difference between the channel gains of different clusters both increases with $M$. It can also be remarked that, as it is often the case in clustering analysis, the curve forms an "elbow" shape, from $M=2$ to 4 . Hence, according to the elbow method, a suitable value of $M$ can be found within this interval. For instance, a cluster number of $M=3$, which exhibits a $\mathrm{F}$ value of $99 \%$, seems a reasonable value for $M$ in Fig. 2. Note that requirements and constraints (e.g. accommodating more users during peak hours) can also be considered (in conjunction with the elbow method) to determine an appropriate value of $M$; i.e. first use the elbow method to find a suitable range of $M$ values and then select an appropriate value of $M$ within this range based on specific 


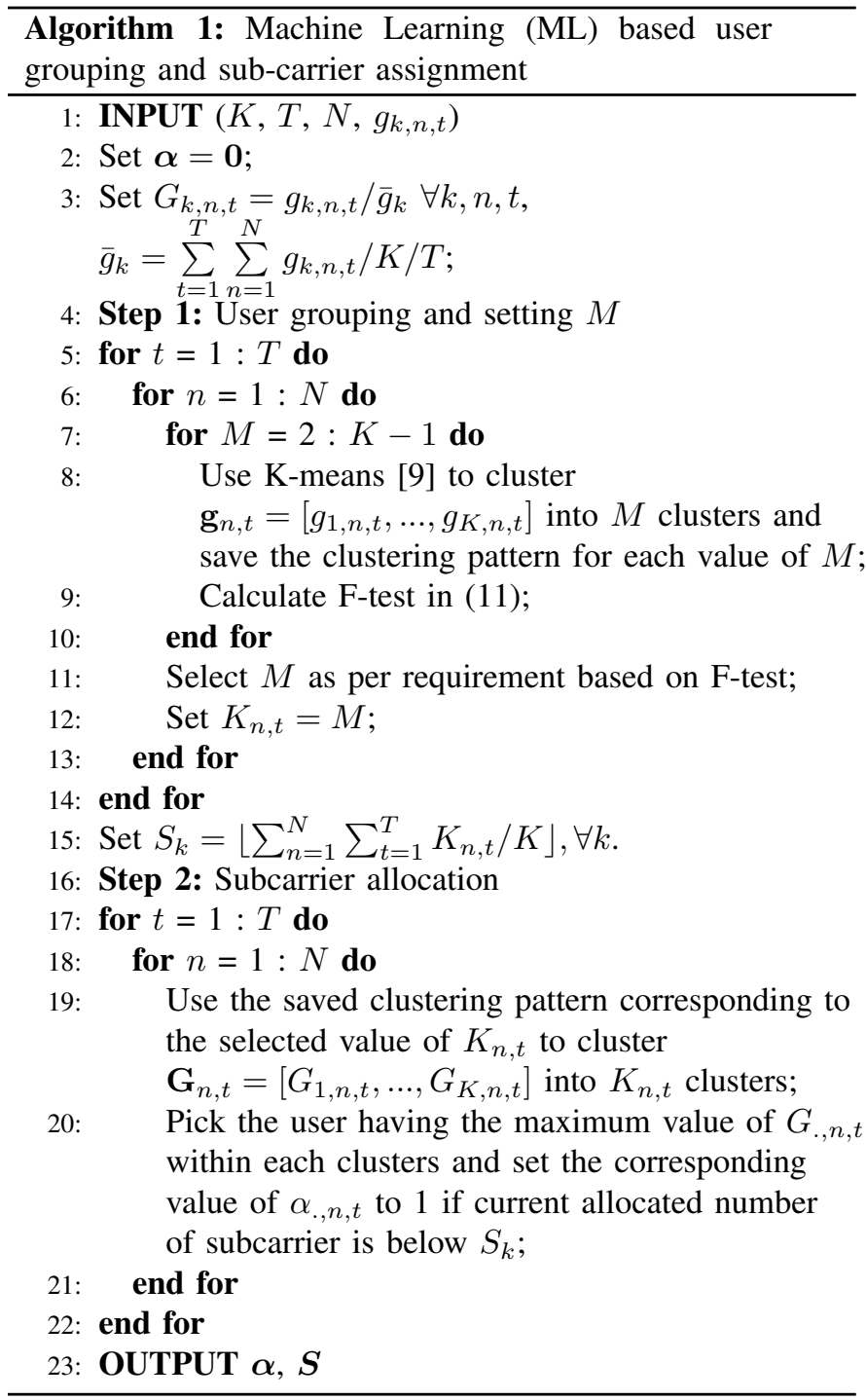

requirements/constraints.

Once a suitable value of $M$ has been chosen for a given subcarrier $n$ at time slot $t$, a user of each cluster is scheduled on this subcarrier/time slot, as further detailed in Algorithm 1. Hence, the number of users that are grouped on this subcarrier/time slot is $K_{n, t}=M$. In Algorithm 1, $G_{k, n, t}$, which is used to perform the subcarrier allocation, is the normalized channel gain. We use $G_{k, n, t}$ instead of $g_{k, n, t}$ in the subcarrier allocation to remain fair with all the users and to minimize the EMF exposure, by avoiding the worst subcarrier allocation to the user over a given time slot $t$. In addition, $S_{k}$ is the number of allocated subcarriers per user.

\section{Power Assignment}

Following the user grouping and subcarrier allocation process of Section III, an optimum amount of transmit power must be allocated to each user, in order to minimize the uplink EMF-exposure. We know from section II-B that, even when $\alpha$ is known (from the subcarrier allocation process), the problem in (9) and (10) is non-convex due to the non-linearity of constraint (10a). By applying the following change of variables (based on a simple transformation of (5)):

$$
p_{k, n, t}=\frac{\left(2^{r_{k, n, t}}-1\right)\left(\sigma^{2}+I_{k, n, t}\right)}{g_{k, n, t}},
$$

and by substituting $p_{k, n, t}$ by (12) in (9) and (10), this optimization problem can be re-expressed as:

$\min _{\boldsymbol{r}} \frac{\tau}{T} \sum_{k=1}^{K}\left(\bar{p}_{k}(T)+\sum_{t=1}^{T} \sum_{n=1}^{N} \frac{\alpha_{k, n, t}\left(2^{r_{k, n, t}}-1\right)\left(\sigma^{2}+I_{k, n, t}\right)}{g_{k, n, t}}\right)$,

subject to:

$$
\begin{aligned}
& C_{1}: w \tau \sum_{t=1}^{T} \sum_{n=1}^{N} \alpha_{k, n, t} r_{k, n, t}=B r_{k} \\
& C_{2}: \sum_{n=1}^{N} \frac{\alpha_{k, n, t}\left(2^{r_{k, n, t}}-1\right)\left(\sigma^{2}+I_{k, n, t}\right)}{g_{k, n, t}} \leq P_{k}^{\max }, \\
& C_{3}: \alpha_{k, n, t}\left(\left(2^{r_{k, n, t}}-1\right)\left(\sigma^{2}+I_{k, n, t}\right) / I_{k, n, t}\right) \geq \zeta,
\end{aligned}
$$

where $r_{k, n, t}=b_{k, n, t} /(w \tau)$ represents the spectral efficiency of user $k$ on subcarrier $n$ during time slot $t$, and $\mathbf{r}=\left[r_{1,1,1} ; \ldots ; r_{K, N, 1} ; r_{1,1,2} ; \ldots ; r_{K, N, T}\right]$. Contrary to (10), the constraint $C_{1}$ in (13) is an affine function of $\mathbf{r}$. Moreover, the objective function and the constrained $C_{2}$ and $C_{3}$ are all convex functions of $\mathbf{r}$ in (13), when assuming that $I_{k, n, t}$ is fixed. Thus, (13) is a convex optimization problem and its Lagrangian can be defined as:

$$
\begin{aligned}
& \mathcal{L}\left(r_{k, t}, \lambda_{k}, \mu_{k, t}, \delta_{k, n, t}\right)= \\
& \frac{\tau}{T} \sum_{k=1}^{K}\left(\bar{p}_{k}+\sum_{t=1}^{T} \sum_{n=1}^{N} \frac{\alpha_{k, n, t}\left(2^{r_{k, n, t}}-1\right)\left(\sigma^{2}+I_{k, n, t}\right)}{g_{k, n, t}}\right) \\
& +\lambda_{k}\left(B r_{k}-w \tau \sum_{t=1}^{T} \sum_{n=1}^{N} \alpha_{k, n, t} r_{k, n, t}\right) \\
& +\mu_{k, t}\left(P_{k}^{\max } g_{k, n, t}-\sum_{n=1}^{N} \alpha_{k, n, t}\left(2^{r_{k, n, t}}-1\right)\left(\sigma^{2}+I_{k, n, t}\right)\right) \\
& +\delta_{k, n, t} \alpha_{k, n, t}\left(\zeta-\left(2^{r_{k, n, t}}-1\right)\left(\sigma^{2}+I_{k, n, t}\right) / I_{k, n, t}\right) ;
\end{aligned}
$$

here, $\lambda_{k}, \mu_{k, t}$ and $\delta_{k, n, t}$ are the Lagrange multipliers being associated with constraints $C_{1}, C_{2}$ and $C_{3}$, respectively.

By solving $\nabla \mathcal{L}\left(r_{k, t}, \lambda_{k}, \mu_{k, t}, \delta_{k, n, t}\right)=0$, and ensuring that all the Karush-Kuhn-Tucker (KKT) conditions [29] are met, we obtain (See Appendix for the proof):

$$
r_{k, n, t}=\max \left(0, \log _{2} \chi+\log _{2}\left(\frac{w g_{k, n, t}}{\ln (2)\left(\sigma^{2}+I_{k, n, t}\right)}\right)\right)
$$

where, $\chi$ can be represented as:

$$
\chi=\frac{\lambda_{k}^{\star}}{\left(1 / T-\left(\mu_{k, t}^{\star}+\delta_{k, n, t}^{\star} g_{k, n, t} / I_{k, n, t}\right) / \tau\right)} .
$$

Equation (16) is a water-filling type of solution that can be evaluated by using iterative methods, e.g. Secant and NewtonRaphson [36]. We then follow a two-stage strategy to perform 


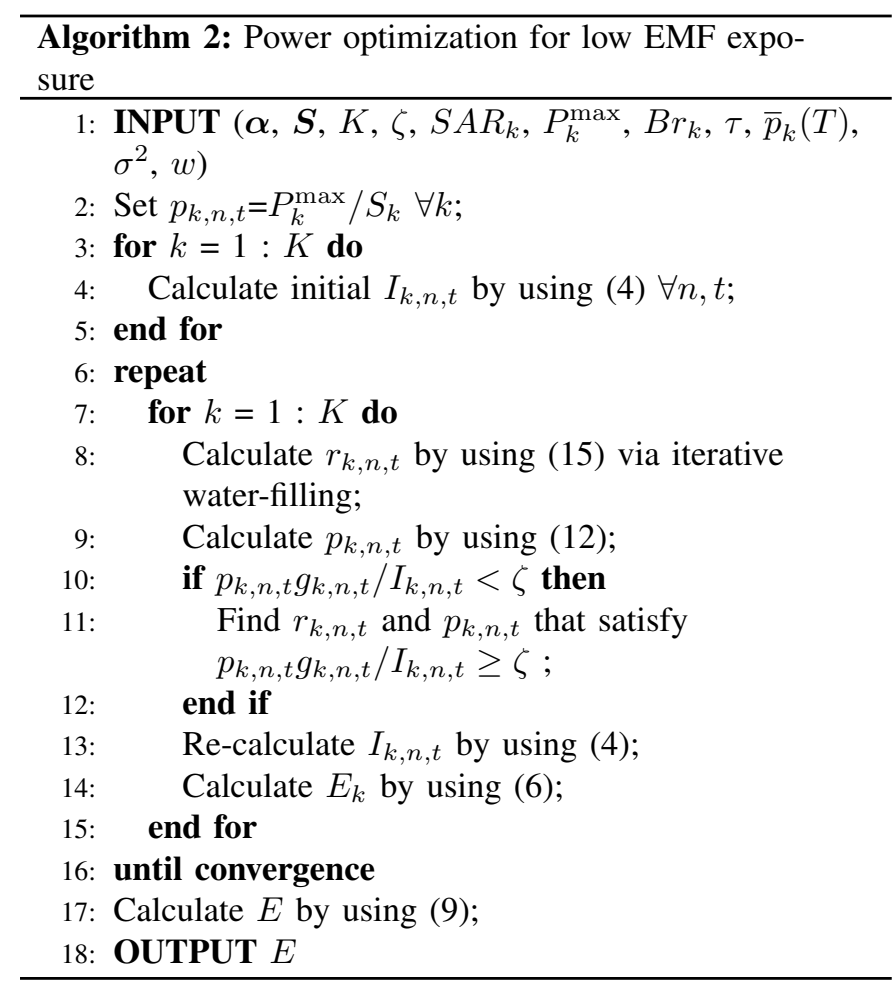

the power allocation. Firstly, the transmit power of each user is equally divided between its $S_{k}$ subcarriers by averaging it over the maximum power level of each UE. In turn, this is used to calculate the initial interference levels for each group of users sharing the same subcarrier. The interference is dependent on the SIC process, and its outcome is itself dependent of the reference threshold $\zeta$ (see equation (3)). As it is mentioned in Section II. A., the SIC process at the BS would decode the user with the best channel gain first (when assuming equal transmit power), i.e. in descending order: $g_{\pi(1), n, t} \geq g_{\pi(2), n, t}, \geq \ldots ., \geq$ $g_{\pi\left(K_{n, t}\right), n, t}$ such that the user with the worst channel gain will be decoded as free from interference if $C_{3}$ in (13), which is dependent of $\zeta$, is always met. Secondly, iterative waterfilling is used to optimize the power levels of each $k$ user, allocated to the same subcarrier $n$, while satisfying the QoS constraint $B r_{k}$ and transmit power constraint $P_{k}^{\max }$ for each user. The power level must also comply with constraint $C_{3}$ and if it is not the case, it is then recomputed. The two-stage process is then repeated until convergence, i.e. until the different values of $p_{k, n, t}$ remain very similar between two iterations of this process. More details about the power allocation algorithm are provided in Algorithm 2.

\section{Numerical ANALYSis}

In this section, the performance of our proposed ML based subcarrier allocation and power optimization for a PD-NOMA system is validated by using MATLAB simulations. We have considered a single cell of radius $R$, where the $\mathrm{BS}$ is located in the centre of the cell (as in Fig. 1) and $K$ users are randomly placed uniformly within this cell. We have modeled the propagation effects between any user and the BS by using the path-loss model defined in [35] (see page 378)
TABLE II

DEFAULT SIMULATION PARAMETERS

\begin{tabular}{|l||l|}
\hline \hline \multicolumn{1}{|c|}{ Symbol } & \multicolumn{1}{c|}{ Meaning } \\
\hline \hline$R$ & 500 meters \\
\hline$P_{o}$ & $-112 \mathrm{dBm}$ \\
\hline$\tau$ & $1 \mathrm{~ms}$ \\
\hline$P^{\text {ref }}$ & $1 \mathrm{~W}$ \\
\hline$K$ & 15 \\
\hline$W$ & $10 \mathrm{MHz}$ \\
\hline$T$ & 10 \\
\hline$N$ & 128 \\
\hline$P_{k}^{\max }$ & $0.2 \mathrm{~W}$ \\
\hline$\sigma^{2}$ & $-174 \mathrm{dBm} / \mathrm{Hz}$ \\
\hline $\mathrm{SAR}_{k}$ & $1 \mathrm{~W} / \mathrm{Kg}$ \\
\hline $\mathrm{SAR}_{k}^{a}$ & $0.658545 \mathrm{~W} / \mathrm{Kg}$ \\
\hline $\mathrm{SAR}_{k}^{b}$ & $0.561139 \mathrm{~W} / \mathrm{Kg}$ \\
\hline$a$ & $4 \mathrm{bits}$ \\
\hline \hline
\end{tabular}

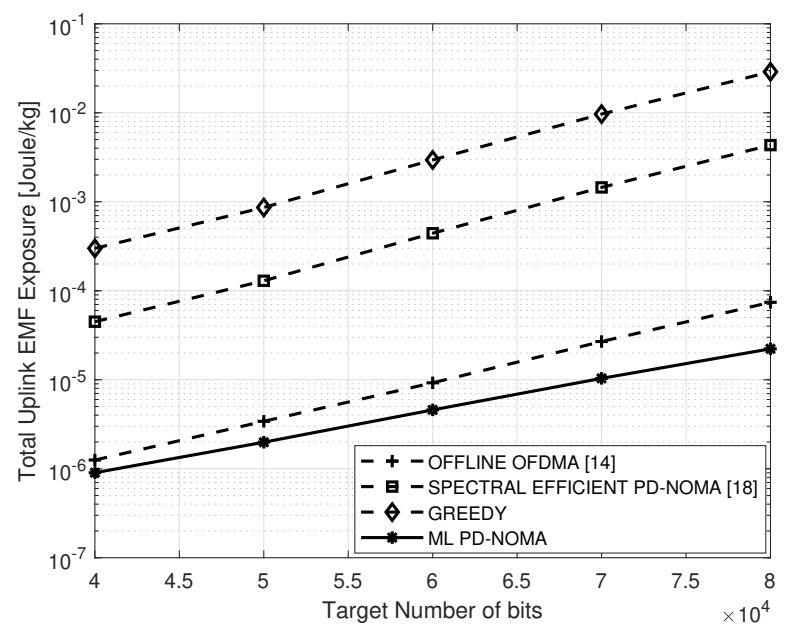

Fig. 3. The comparison of total EMF uplink exposure vs. the target number of bits for a fixed $K=15$ users, $T=10$ time slots, and $N=128$ subcarriers.

in conjunction with Rayleigh fading. The detailed simulation parameters are listed in Table II. We have assumed that all the users in the cell uses similar devices that obeys the Federal communications commission (FCC) guidelines, i.e. $\mathrm{SAR}_{k}$ and $P^{\text {ref }}$ are constants. We have also assumed that all the users have the same target number of bits, i.e., $B r_{1}=B r_{2}=\ldots=B r_{K}$. In addition, we have set $\zeta=1$ in our simulation. In our simulation setup, we first use Algorithm 1 to group the users and perform the subcarrier allocation before feeding its output to Algorithm 2, for dynamically adjusting the transmit power of each subcarrier in a low-exposure manner.

\section{A. Simulation Results}

In Fig. 3, we compared our proposed technique with the offline scheme of [14], as well as the spectral efficient scheme of [18], and the greedy scheme by varying the number of target bits for $T=10$ time slots, $K=15$ users and for 


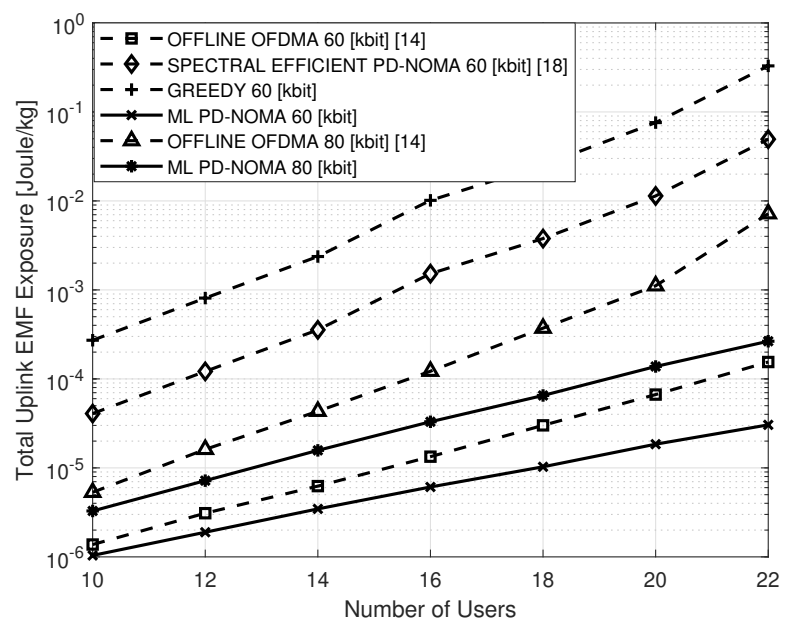

Fig. 4. The comparison of total EMF uplink exposure vs. varying the number of user for different target number of bits and for a fixed $T=10$ time slots.

$N=128$ subcarriers. It is noted that in greedy scheme, the criteria for the subcarrier allocation is based on the user having the best channel characteristics on each subcarrier per time slot. It is quite evident that the total EMF-exposure for the uplink communication increases as the target number of bits increases, since for a fixed $T$ and $N$ values each user requires more power to transmit more information. In comparison with the classic EMF-aware OFDMA scheme of [14], our proposed ML PD-NOMA based strategy achieves an exposure reduction of $28 \%$ for $\mathrm{Br}=40 \mathrm{kbit}$, and even $50 \%$ for $\mathrm{Br}=60 \mathrm{kbit}$. This reduction in EMF exposure can be explained from the fact that our scheme, which uses PD-NOMA, can allow a maximum of $K_{n, t}$ users to use the same subcarrier, hence improving the spectral efficiency, while comparing with the OFDMA counterpart of [14]. Moreover, the use of SIC as a decoding strategy at the receiver end also helps to reduce the total uplink EMF exposure by allowing the user with the worst channel gain to transmit its data free of interference, hence minimizing its total transmit power. While comparing our proposed ML PD-NOMA based strategy with the spectral efficient PD-NOMA of [18] and the greedy scheme, our scheme reduces the EMF exposure by at least 2 and 3 order of magnitude for $\mathrm{Br}=40 \mathrm{kbit}$. In [18], as the system tries to maximize the weighted sum-rate of $K$ users over each $t$ time slot, an increase in EMF exposure is observed, while comparing with our proposed ML PD-NOMA based strategy and [14]. Furthermore, the greedy scheme has the highest value of total EMF exposure, as on each subcarrier it uses all of the available transmit power in an attempt to maximize the transmitted number of bits.

In order to further confirm the effectiveness of our proposed scheme as compared to the state-of-the-art techniques in [14], [18], and the greedy scheme, we compare in Fig. 4 these four schemes for a varying value of $K$ when the target number of bits is either fixed to 60 or $80 \mathrm{kbit}$. The total EMF exposure for the uplink communication increases with the number of users since more transmission power is required for each user to transmit the same amount of information when the

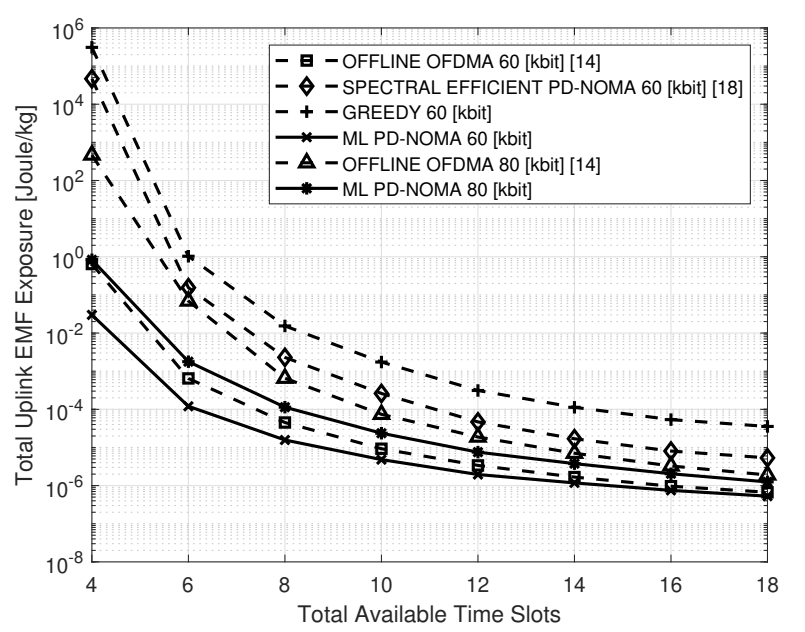

Fig. 5. The comparison of total EMF uplink exposure vs. varying the time slots for different target number of bits and for a fixed $K=15$ users.

number of subcarriers and the transmission window are fixed. Accordingly, the number of subcarriers allocated to each user decreases with an increase in the number of users, as the value of $S_{k}$ depends on the number of users, hence the overall EMF exposure increases. In comparison with [14], our scheme shows a stable trend, as PD-NOMA allows each subcarrier to be shared by a maximum of $K_{n, t}$, hence providing a reduction of at least $25 \%$ in the total EMF exposure for a fixed $B r=60$ kbit. For $B r=80 \mathrm{kbit}$, our proposed scheme reduces the EMF exposure by at least $38 \%$, while comparing it to the offline scheme of [14]. Moreover, while comparing the proposed ML PD-NOMA based strategy with spectral efficient PDNOMA of [18] and the greedy scheme, our scheme reduces the EMF-exposure by at least 2 and 3 order of magnitude for $B r=60 \mathrm{kbit}$. It can also be remarked that the gap of performance between the schemes increases as the number of users increases. For instance, our scheme can accommodate 4 more users (22 instead of 18) than the scheme of [14] for the same total exposure of $3 \times 10^{-5} \mathrm{~J} / \mathrm{Kg}$.

In Fig. 5, we study the performance of our scheme against [14], [18] and the greedy scheme, respectively, for a varying number of time slots and by fixing the value of $K$ and $N$, when the target number of bits is either fixed to 60 or $80 \mathrm{kbit}$. It can be inferred from Fig. 5, that with an increase in the number of time slots, the EMF exposure decreases, which can be related to the fact that each user can reduce its transmission power by distributing the target number of bits over the increasing value of $T$. Moreover, in comparison to the offline scheme of [14], our scheme reduces the total EMF exposure. Our proposed scheme reduces the exposure by at least $21 \%$ and $34 \%$ for fixed values of $\mathrm{Br}=60 \mathrm{kbit}$ and $\mathrm{Br}=80 \mathrm{kbit}$, respectively, in comparison with the offline scheme of [14]. This performance improvement can be explained by the fact that the availability of resources is larger in PD-NOMA than in OFDMA. Although, the sharing of a subcarrier by $K_{n, t}$ users imposes an additional interference, in addition to the noise, yet the usage of SIC, as a decoding technique at the receiver end, allows to mitigate its effect. In comparison to [18] and greedy 


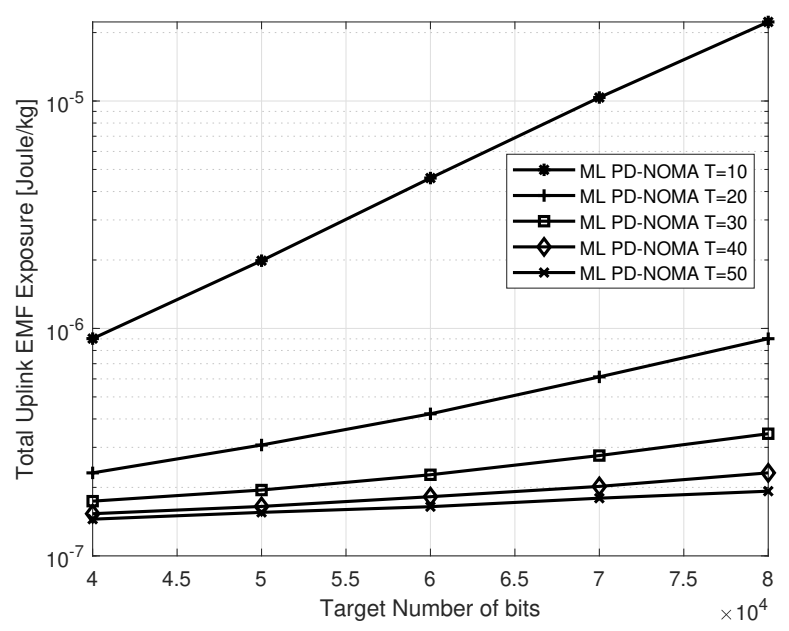

Fig. 6. The comparison of total EMF uplink exposure vs. varying the target number of bits for different values of $T$ and for a fixed $K=15$ users.

scheme, our scheme reduces the EMF-exposure by at least 1 and 2 order of magnitude for $\mathrm{Br}=60 \mathrm{kbit}$. To further validate the variation of the total uplink EMF exposure on increasing the number of time slots over varying target number of bits, in Fig. 6, we have studied the effect of increasing the value of $T$ on the total EMF exposure for a fixed value of $K$ and $N$. It is evident and supports our results in Fig. 5 that with an increase in the number of time slots, the EMF exposure decreases, which can be related to the fact that each user can reduce its transmission power by distributing the target number of bits over an increasing value of $T$.

\section{B. Scheme Validity for Real Applications}

In this section, we have considered a PIFA, having one transmit antenna available for the uplink transmission, and radiating at a resonance frequency of $2.1 \mathrm{GHz}$, to calculate the value of the SAR from simulation results. As per IEEE recommendations [37], we have considered two scenarios using the Voxel models (heterogeneous human biological replication) available in computer simulation technology (CST) 2018, to calculate the maximum SAR value for $1 \mathrm{~g}$ of body mass. Fig. 7 illustrates these two scenarios: (a) being the cheek position, and (b) is the tilt position. We have used IEEE/IEC 62704-1 averaging method [38] to measure the maximum SAR value, while employing the maximum transmit power $P_{k}^{\max }=0.2 \mathrm{~W}$. The SAR values obtained from these simulations are listed as $\mathrm{SAR}_{k}^{a}$ and $\mathrm{SAR}_{k}^{b}$ in Table II. In Fig. 8, we show the variation of the total uplink EMF exposure as a function of the number of bits by using the $\mathrm{SAR}_{k}^{a}$ (for cheek position), $\mathrm{SAR}_{k}^{b}$ (for tilt position) and normalized $\mathrm{SAR}_{k}$ values. The results obtained in Fig. 8 shows a similar trend for three cases, but the values of SAR obtained using experimental analysis produce a reduction in the total uplink EMF exposure, which can be justified by the lower calculated values of maximum SAR for the two scenarios. Furthermore, the low SAR values further justifies the suitability of using a PIFA in mobile handsets.

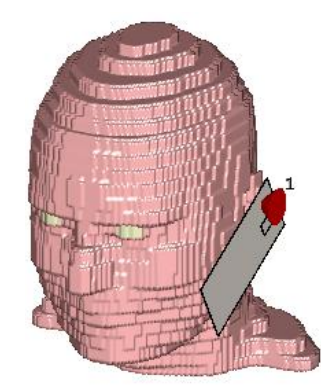

(a)

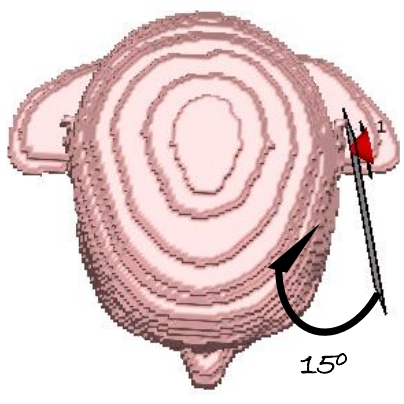

(b)

Fig. 7. Illustration of SAR testing positions (a) Cheek position, (b) Tilt position [37]

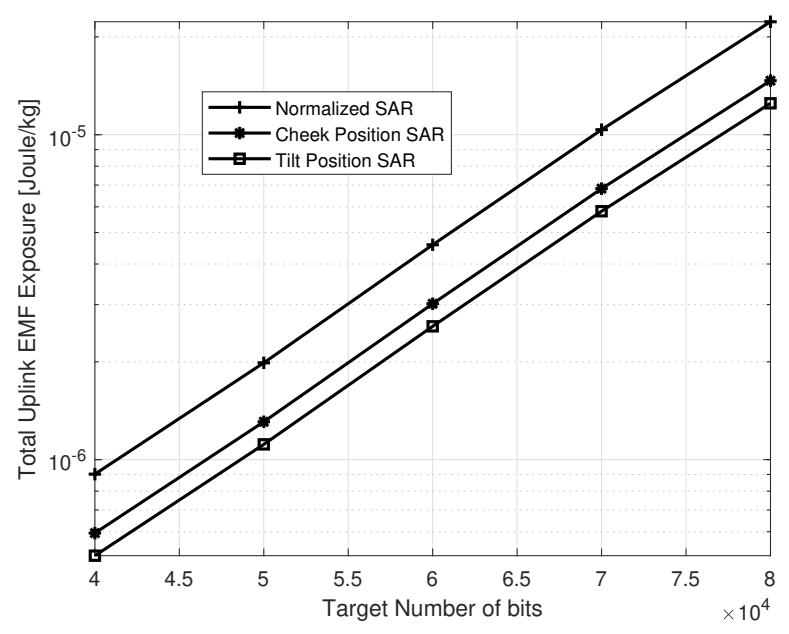

Fig. 8. Comparison of total uplink exposure with target number of bits for different SAR values.

\section{CONCLUSiON}

In this paper, we have proposed a ML based user grouping and resource allocation strategy to minimize the EMF exposure for the uplink of PD-NOMA systems. Firstly, by using the ML based user grouping and resource allocation algorithm, we intelligently group the users into different clusters and performs subcarrier allocation. Secondly, using the allocated subcarriers, we then perform power assignment, by applying a change in variables to formulate a convex optimization problem. In comparison, to the state-of-the-art techniques, our 
scheme reduces the EMF exposure by a fair margin. Moreover, we have also included the SAR values, calculated by using the simulation scenarios based on IEEE recommendations. By using a PIFA equipped with a single antenna, we have shown that our scheme can still reduce total EMF exposure, and have also justified the suitability of using a PIFA in mobile handsets. In future we are aiming to extend our work to multi-antenna systems.

\section{APPENDIX}

$$
\begin{aligned}
& \nabla \mathcal{L}\left(r_{k, t}, \lambda_{k}, \mu_{k, t}, \delta_{k, n, t}\right)=0 \text { is equivalent to } \\
& \frac{\tau}{T} \alpha_{k, n, t}\left(\frac{\ln (2) 2^{r_{k, n, t}}\left(\sigma^{2}+I_{k, n, t}\right)}{g_{k, n, t}}\right)-\lambda_{k} \alpha_{k, n, t} w \tau \\
& -\alpha_{k, n, t}\left(\frac{\ln (2) \mu_{k, t} 2^{r_{k, n, t}}\left(\sigma^{2}+I_{k, n, t}\right)}{g_{k, n, t}}\right) \\
& -\alpha_{k, n, t}\left(\frac{2^{r_{k, n, t}} \ln (2) \delta_{k, n, t}\left(\sigma^{2}+I_{k, n, t}\right)}{I_{k, n, t}}\right)=0,
\end{aligned}
$$

Then, equation (17) can further be rearranged as

$$
\begin{aligned}
& 2^{r_{k, n, t}} \ln (2)\left(\sigma^{2}+I_{k, n, t}\right) / g_{k, n, t} \\
& \left(\tau / T-\mu_{k, t}-\delta_{k, n, t} g_{k, n, t} / I_{k, n, t}\right)=\omega \tau \lambda_{k} .
\end{aligned}
$$

Equation (18) can then be re-expressed as

$$
2^{r_{k, n, t}}=\frac{\tau \lambda_{k}}{\tau / T-\mu_{k, t}-\delta_{k, n, t} g_{k, n, t} / I_{k, n, t}} \frac{\omega g_{k, n, t}}{\ln (2)\left(\sigma^{2}+I_{k, n, t}\right)},
$$

which finally leads to the result in (15) by applying further simplifications to (19).

\section{REFERENCES}

[1] F. Boccardi, R. W. Heath, A. Lozano, T. L. Marzetta, and P. Popovski, "Five disruptive technology directions for 5G," IEEE communications magazine, vol. 52, no. 2, pp. 74-80, 2014.

[2] I. A. for Research on Cancer et al., "IARC classifies radiofrequency electromagnetic fields as possibly carcinogenic to humans," Press release, no. 208, 2011.

[3] L. O. Hardell, M. Carlberg, F. Söderqvist, K. H. Mild, and L. L. Morgan, "Long-term use of cellular phones and brain tumours-increased risk associated with use for $>10$ years," Occupational and environmental medicine, 2007.

[4] Radiofrequency Electromagnetic Fields, "Evaluating compliance with FCC guidelines for human exposure to radio frequency electromagnetic fields," OET Bull, vol. 65, pp. 1-53, 1997.

[5] ICNIRP, "GUIDELINES FOR LIMITING EXPOSURE TO TIME-VARYING ELECTRIC, MAGNETIC AND ELECTROMAGNETIC FIELDS $(100 \mathrm{kHz}$ TO $300 \quad \mathrm{GHz})$. https://www.icnirp.org/cms/upload/consultation-upload/ICNIRP-RFGuidelines-PCD-2018-07-11.pdf. Accessed: 2019-02-26.

[6] F. C. Commission et al., "Specific absorption rate (SAR) for cell phones: what it means for you," 2014.

[7] L. Dai, B. Wang, Z. Ding, Z. Wang, S. Chen, and L. Hanzo, "A Survey of Non-Orthogonal Multiple Access for 5G," IEEE Communications Surveys \& Tutorials, 2018.

[8] S. Mounchili and S. Hamouda, "Pairing Distance Resolution and Power Control for Massive Connectivity Improvement in NOMA Systems," IEEE Transactions on Vehicular Technology, vol. 69, no. 4, pp. 40934103, 2020.

[9] J. Cui, Z. Ding, P. Fan, and N. Al-Dhahir, "Unsupervised machine learning-based user clustering in millimeter-wave-NOMA systems," IEEE Transactions on Wireless Communications, vol. 17, no. 11, pp. 7425-7440, 2018.
[10] H. Nikopour, E. Yi, A. Bayesteh, K. Au, M. Hawryluck, H. Baligh, and J. Ma, "Scma for downlink multiple access of $5 \mathrm{~g}$ wireless networks," in 2014 IEEE global communications conference, pp. 3940-3945, IEEE, 2014.

[11] D. Cai, P. Fan, and P. T. Mathiopoulos, "A tight lower bound for the symbol error performance of the uplink sparse code multiple access," IEEE Wireless Communications Letters, vol. 6, no. 2, pp. 190-193, 2017.

[12] M. A. Jamshed, F. Heliot, and T. Brown, "A Survey on Electromagnetic Risk Assessment and Evaluation Mechanism for Future Wireless Communication Systems," IEEE Journal of Electromagnetics, RF and Microwaves in Medicine and Biology, 2019.

[13] M. T. Islam, M. R. I. Faruque, and N. Misran, "Reduction of specific absorption rate (SAR) in the human head with ferrite material and metamaterial," Progress In Electromagnetics Research, vol. 9, pp. 4758, 2009.

[14] Y. A. Sambo, M. Al-Imari, F. Héliot, and M. A. Imran, "Electromagnetic emission-aware schedulers for the uplink of OFDM wireless communication systems," IEEE Transactions on Vehicular Technology, vol. 66, no. 2, pp. 1313-1323, 2016.

[15] Y. A. Sambo, F. Heliot, and M. A. Imran, "Electromagnetic EmissionAware Scheduling for the Uplink of Multicell OFDM Wireless Systems," IEEE Transactions on Vehicular Technology, vol. 66, no. 9, pp. 82128222, 2017.

[16] A. De Domenico, L. F. Díez, R. Agüero, D. Kténas, and V. Savin, "EMF-Aware Cell Selection in Heterogeneous Cellular Networks.," IEEE Communications Letters, vol. 19, no. 2, pp. 271-274, 2015.

[17] H. Zuo and X. Tao, "Power allocation optimization for uplink nonorthogonal multiple access systems," in Wireless Communications and Signal Processing (WCSP), 2017 9th International Conference on, pp. 15, IEEE, 2017.

[18] M. Al-Imari, P. Xiao, and M. A. Imran, "Receiver and resource allocation optimization for uplink NOMA in 5G wireless networks," in Wireless Communication Systems (ISWCS), 2015 International Symposium on, pp. 151-155, IEEE, 2015.

[19] Y. Yin, Y. Peng, M. Liu, J. Yang, and G. Gui, "Dynamic user groupingbased NOMA over Rayleigh fading channels," IEEE Access, vol. 7, pp. 110964-110971, 2019.

[20] A. K. Jain, "Data clustering: 50 years beyond K-means," Pattern recognition letters, vol. 31, no. 8, pp. 651-666, 2010

[21] K. Bindra, A. Mishra, et al., "Effective Data Clustering Algorithms," in Soft Computing: Theories and Applications, pp. 419-432, Springer, 2019.

[22] B. Di, L. Song, and Y. Li, "Sub-channel assignment, power allocation, and user scheduling for non-orthogonal multiple access networks," IEEE Transactions on Wireless Communications, vol. 15, no. 11, pp. 76867698, 2016.

[23] M. A. Jamshed, O. Amjad, F. Heliot, and T. Brown, "EMF-reduction Uplink Resource Allocation Scheme for Non-Orthogonal Multiple Access Systems," in 2019 IEEE Wireless Communications and Networking Conference Workshop (WCNCW), pp. 1-5, IEEE, 2019.

[24] G. D. Golden, C. Foschini, R. A. Valenzuela, and P. W. Wolniansky, "Detection algorithm and initial laboratory results using V-BLAST space-time communication architecture," Electronics letters, vol. 35, no. 1 , pp. 14-16, 1999.

[25] Z. Ali, G. A. S. Sidhu, M. Waqas, and F. Gao, "On fair power optimization in nonorthogonal multiple access multiuser networks," Transactions on Emerging Telecommunications Technologies, vol. 29, no. 12, p. e3540, 2018.

[26] E. Conil, "D2. 4 Global wireless exposure metric definition v1," LexNet project, 2013.

[27] F. C. Commission et al., "Evaluating Compliance with FCC Guidelines for Human Exposure to Radiofrequency Electromagnetic Fields, Supplement C," OET Bulletin, vol. 65, pp. 1-57.

[28] 3GPP, "3GPP TS 36.213, 'Evolved Universal Terrestrial Radio Access (E-UTRA); Physical layer procedures (Release 12),” 2015.

[29] Z.-Q. Luo and W. Yu, "An introduction to convex optimization for communications and signal processing," IEEE Journal on selected areas in communications, vol. 24, no. 8, pp. 1426-1438, 2006.

[30] M. Al-Imari, P. Xiao, M. A. Imran, and R. Tafazolli, "Low complexity subcarrier and power allocation algorithm for uplink OFDMA systems," EURASIP Journal on Wireless Communications and Networking, vol. 2013, no. 1, pp. 1-6, 2013.

[31] H. Ye, G. Y. Li, and B.-H. F. Juang, "Deep reinforcement learning based resource allocation for V2V communications," IEEE Transactions on Vehicular Technology, vol. 68, no. 4, pp. 3163-3173, 2019.

[32] R. Tibshirani, G. Walther, and T. Hastie, "Estimating the number of clusters in a data set via the gap statistic," Journal of the Royal Statistical 
Society: Series B (Statistical Methodology), vol. 63, no. 2, pp. 411-423, 2001.

[33] C. Goutte, P. Toft, E. Rostrup, F. A. Nielsen, and L. K. Hansen, "On clustering fMRI time series," NeuroImage, vol. 9, no. 3, pp. 298-310, 1999.

[34] C. Dougherty, Introduction to econometrics. Oxford University Press, 2011.

[35] J. Wu, S. Rangan, and H. Zhang, Green communications: theoretical fundamentals, algorithms, and applications. CRC press, 2016.

[36] M. Abramowitz and I. Stegun, "Handbook of Mathematical Functions: With Formulas, Graphs, and Mathematical Tables Applied mathematics series," National Bureau of Standards, Washington, DC, 1964.

[37] "IEEE Recommended Practice for Determining the Peak SpatialAverage Specific Absorption Rate (SAR) in the Human Head from Wireless Communications Devices: Measurement Techniques," IEEE Std 1528-2013 (Revision of IEEE Std 1528-2003), pp. 1-246, Sep. 2013.

[38] "IEC/IEEE International Standard - Determining the peak spatialaverage specific absorption rate (SAR) in the human body from wireless communications devices, $30 \mathrm{MHz}$ to $6 \mathrm{GHz}$ - Part 1: General requirements for using the finite-difference time-domain (FDTD) method for SAR calculations," IEC/IEEE 62704-1:2017, pp. 1-86, Oct 2017.

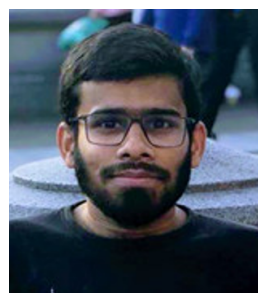

Muhammad Ali Jamshed (S'19) received the B.Sc. degree in electrical engineering from COMSATS University, Islamabad, Pakistan, in 2013 and the M.Sc. degree in Wireless communications from the Institute of Space Technology, Islamabad Pakistan, in 2016, and Ph.D. degree from University of Surrey, Guildford, U.K, in 2021. He was nominated for Departmental Prize for Excellence in Research in 2019 at the University of Surrey. His main research interests include EMF exposure reduction, low SAR antennas for mobile handsets, machine learning for wireless communication, Backscatter communication, and wireless sensor networks. He is serving as a Reviewer for Transactions on Emerging Telecommunications Technologies, Wiley. Moreover, he served as a Reviewer, and the Session Chair, at a number of well-known conferences, i.e, ICC, WCNC, VTC, GlobeCom etc. and other scientific workshops.

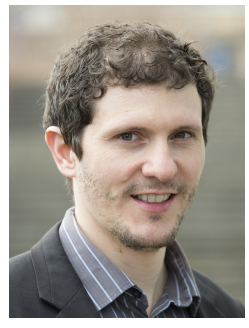

Dr Fabien Héliot is a Lecturer in wireless communications at the Institute for Communication Systems (ICS) of the University of Surrey. He published 100+ peer-reviewed papers, book chapters and technical reports in diverse areas of communications theory and signal processing for wireless communications. He has lead the EPRSC funded project "Electromagnetic field exposure reduction/avoidance for the next generations of wireless communication systems" EP/P005853/1 and has been actively involved (tasks leader) in other funded UK/EU projects related to the topic of this proposal, e.g. metrology for RF EXposure from 5G base station (5GRFEX), Low Exposure Future Networks (LExNet), and Energy Aware Radio and neTwork tecHnologies (EARTH).

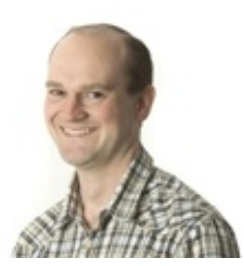

T im W. C. Brown (S'00-M'04) graduated from the University of Surrey, UK, in 1999 with a BEng in Electronic Engineering and since graduated with a $\mathrm{PhD}$ in antenna diversity for mobile terminals in the Centre for Communication Systems Research (CCSR) [Now named the Institute for Communications Systems] in 2004. Since completing his doctoral research, he has continued his research interests in antennas, propagation and radio frequency (RF) engineering. This has included postdoctoral research from 2004-2006 at Aalborg University, Denmark and his present post as an associate professor in RF, Antennas and Propagation at the University of Surrey. His research interests include multi antenna technologies both for capacity enhancement but also ultra low exposure devices and wireless networks. 